\title{
The Role of Selected Environmental Bacteria in Decomposition of Nitrofuran Antibiotics
}

\author{
Amanda Pacholak, Ewa Kaczorek \\ Institute of Chemical Technology and Engineering, Poznan University of Technology \\ Berdychowo 4, 60-965 Poznan, Poland \\ amanda.d.pacholak@doctorate.put.poznan.pl; ewa.kaczorek@put.poznan.pl
}

\section{Extended Abstract}

Pharmaceuticals used in human and veterinary medicine belong to the emerging environmental contaminants. A special class of these pollutants are antimicrobial agents. Their undesirable presence in the natural environmental compartments may induce the antibiotics resistance in pathogenic bacteria and have an adverse effect on natural microbial communities. One group of the broad-spectrum antimicrobial agents are nitrofuran derivatives [1], [2]. These drugs had been widely applied as the growth promoters in livestock production till 1995 when their use in the livestock production was limited in the European Union [3]. However, nitrofurans (NFs) are still easily available and used in the treatment of bacterial and protozoan infections. There is a high probability of the natural environment contamination with these pollutants. There are several studies describing the physicochemical methods in the removal of NFs [3], [4]. However, their potential application is limited and requires the specialised equipment. Hence, the need for closer understanding of these compounds influence on environmental bacteria, and their degradation is of a great importance.

The principal aim of the research is to investigate the biodegradation of nitrofuran derived drugs by newly isolated environmental bacterial strains. Moreover, the specific objectives are to determine the changes in the microbial cell properties and enzymatic activity after exposure to selected nitrofuran-derived drugs.

The scope of the research presented includes, among others, determination of biodegradation of selected nitrofurans with the use of HPLC-MS/MS, analysis of changes in cell surface hydrophobicity, total and inner membrane permeability of microbial strains degrading compounds tested as well as examination of metabolic activity and glutathione $\mathrm{S}$ transferase (GST) activity of microbes subjected to exposure to selected nitrofurans.

The results show that some of the isolated bacterial strains are able to decompose selected nitrofuran derivatives. Importantly, a rapid increase in nitrofurantoin degradation was usually noted in the second day of cultivation, e.g. $85 \%$ for S. marcescens, $50 \%$ for S. maltophilia and $70 \%$ for $R$. radiobacter. The final degradation (measured after 28 days) for the strains in question was 95\%,78\% and 85\%, respectively. Moreover, for strains showing high degradation, the activity of GST was significantly higher in samples after exposure to nitrofurans, in comparison with the control samples (without nitrofuran addition). Furthermore, the presence of nitrofurantoin provoked changes in microbial cell surface properties. The highest differences were observed in $S$. marcescens (decrease in inner membrane permeability from 0.80 to $0.55 \mu \mathrm{M} \mathrm{min}{ }^{-1}$, total membrane permeability from 21 to $16 \%$, cell surface hydrophobicity from 62 to $23 \%$ ).

The result of the research significantly widen and deepen the current state of knowledge on biological degradation of nitrofuran-derived pharmaceuticals and may be helpful in further designing of bioremediation technologies of such contaminants as nitrofuran derivatives.

This research was funded by National Science Centre, Poland, grant number 2017/27/B/NZ9/01603.

\section{References}

[1] M. Biošić, I. Škorić, J. Beganović, and S. Babić, "Nitrofurantoin hydrolytic degradation in the environment," Chemosphere, vol. 186, pp. 660-668, Nov. 2017.

[2] P. Grenni, V. Ancona, and A. Barra Caracciolo, "Ecological effects of antibiotics on natural ecosystems: A review," Microchemical Journal, vol. 136, pp. 25-39, Jan. 2018. 
[3] D. Kong, B. Liang, H. Yun, J. Ma, Z. Li, A. Wang, N.-Q. Ren, "Electrochemical degradation of nitrofurans furazolidone by cathode: Characterization, pathway and antibacterial activity analysis," Chemical Engineering Journal, vol. 262, pp. 1244-1251, Feb. 2015.

[4] D. Kong, H. Yun, D. Cui, M. Qi, C. Shao, D. Cui, N. Ren, B. Liang, A. Wang, "Response of antimicrobial nitrofurazone-degrading biocathode communities to different cathode potentials," Bioresource Technology, vol. 241, pp. 951-958, Oct. 2017. 